\title{
Teissier's problem on the proportionality of big and nef classes over a compact Kähler manifold
}

\author{
Jixiang Fu and Jian Xiao
}

\begin{abstract}
We solve Teissier's proportionality problem for transcendental nef classes over a compact Kähler manifold, which says that the equality in the Khovanskii-Teissier inequalities holds for a pair of big and nef classes if and only if the two classes are proportional. This result recovers the previous one of Boucksom-Favre-Jonsson for the case of big and nef line bundles over a (complex) projective algebraic manifold.
\end{abstract}

\section{Introduction}

Around the year 1979, inspired by the Aleksandrov-Fenchel inequalities in convex geometry, Khovanskii and Teissier discovered, independently, deep inequalities in algebraic geometry which are now called Khovanskii-Teissier inequalities. These inequalities present a nice relationship between the theory of mixed volumes and algebraic geometry. Their proofs are based on the usual Hodge-Riemann bilinear relations. A natural problem is how to characterize the equality case in these inequalities for a pair of big and nef line bundles; this problem was considered by Teissier [Tei82, Tei88].

In their nice paper [BFJ09], Boucksom, Favre and Jonsson solved this problem, and the answer is that the equality holds if and only if two line bundles are (numerically) proportional. In their paper, they proved an algebro-geometric version of the Diskant inequality in convex geometry following the same strategy of Diskant, which is based on the differentiability of the volume function of convex bodies. To obtain their Diskant inequality, they developed an algebraic construction of the positive intersection products of pseudo-effective classes and used them to prove that the volume function on the big cone of a projective variety is $\mathcal{C}^{1}$-differentiable, expressing its differential as a positive intersection product. Note that their results hold on any complete algebraic variety over an algebraically closed field of characteristic zero. Later, Cutkosky [Cut15] extended these remarkable results to a complete variety over an arbitrary field.

On the other hand, the Khovanskii-Teissier inequalities for transcendental nef classes follow from any of the works [Dem93, DP03], [DN06] and [Gro90]. So a natural question is how to characterize the equality case in this situation. In this note, we give the same answer to this question as in the algebro-geometric case.

Received 9 November 2015, accepted in final form 13 October 2017.

2010 Mathematics Subject Classification 14C20, 32J25, 32Q15.

Keywords: Kähler manifolds, Khovanskii-Teissier inequalities, Teissier's proportionality problem.

This journal is (C) Foundation Compositio Mathematica 2019. This article is distributed with Open Access under the terms of the Creative Commons Attribution Non-Commercial License, which permits non-commercial reuse, distribution, and reproduction in any medium, provided that the original work is properly cited. For commercial re-use, please contact the Foundation Compositio Mathematica.

Fu was supported in part by NSFC 11121101 and NSFC 11421061. Xiao was supported in part by a CSC fellowship and the Institut Fourier. 


\section{J. FU AND J. XIAO}

In [BFJ09] and [Cut15], a key ingredient in the proof of the differentiability theorem of the volume of big line bundles over a projective variety, and thus in the proof of the algebro-geometric version of the Diskant inequality, is the algebraic Morse inequality

$$
\operatorname{vol}(A-B) \geqslant A^{n}-n A^{n-1} \cdot B
$$

for any nef line bundles $A$ and $B$, where $n$ is the variety's dimension. Hence, if one would like to use their methods to extend the results to transcendental classes, the main missing part is the weak transcendental holomorphic Morse inequality. However, up to now, it has not been completely solved yet (see [Xia15,Pop16]). In this note, without using the transcendental version of the Diskant inequality, we can still solve Teissier's proportionality problem for transcendental classes. Thus, our result recovers the previous one of Boucksom-Favre-Jonsson.

Indeed, the key idea in the proof of our main result was hidden in our previous work [FX14]. Moreover, in the effort to get a better understanding of the structure of complete intersection cones, as an immediate corollary of [LX16, Theorem 2.1] (and its proof), it is observed in op. cit. that the equality characterization for a pair of transcendental classes could be extended easily to any number of big and nef classes. To our knowledge, this result seems new in algebraic and Kähler geometry. For the details, we refer the reader to [LX16, Theorem 2.1].

\section{The main theorem}

Let us first recall the definition of nefness and bigness for $(1,1)$-classes on a compact Kähler manifold. Assume that $X$ is an $n$-dimensional compact Kähler manifold with a Kähler metric $\omega$. Let $\alpha \in H_{B C}^{1,1}(X, \mathbb{R})$ be a $(1,1)$ Bott-Chern class. Then $\alpha$ is called nef if for any $\varepsilon>0$, there exists a smooth representative $\alpha_{\varepsilon} \in \alpha$ such that $\alpha_{\varepsilon}>-\varepsilon \omega$. This definition is equivalent to saying that $\alpha$ belongs to the closure of the Kähler cone of $X$, which is denoted by $\overline{\mathcal{K}}$. And $\alpha$ is called big if there exist a positive number $\delta$ and a positive current $T \in \alpha$ such that $T>\delta \omega$ (such a current $T$ is called a Kähler current). This is equivalent to saying that $\alpha$ belongs to the interior of the pseudo-effective cone, which is denoted by $\mathcal{E}^{\circ}$. For more notions, such as the movable cone $\overline{\mathcal{M}}$ in the following theorem, one can see [BDPP13].

Let $\alpha$ be a big class. Recall that the ample locus $\operatorname{Amp}(\alpha)$ is the set of points $x \in X$ such that there is a Kähler current $T_{x} \in \alpha$ with analytic singularities which is smooth near $x$. Indeed, by [Bou04], there exists a Kähler current $T_{\alpha}$ with analytic singularities such that the complement of $\operatorname{Amp}(\alpha)$ is exactly the set of singularities of $T_{\alpha}$. This implies that $\operatorname{Amp}(\alpha)$ must be a Zariskiopen set of $X$.

Theorem 2.1. Assume that $X$ is an n-dimensional compact Kähler manifold. Let $\alpha, \beta \in \overline{\mathcal{K}} \cap \mathcal{E}^{\circ}$ be two big and nef classes. Write $s_{k}:=\alpha^{k} \cdot \beta^{n-k}$. Then the following statements are equivalent:

(1) $s_{k}^{2}=s_{k-1} \cdot s_{k+1}$ for every $1 \leqslant k \leqslant n-1$;

(2) $s_{k}^{n}=s_{0}^{n-k} \cdot s_{n}^{k}$ for every $0 \leqslant k \leqslant n$;

(3) $s_{n-1}^{n}=s_{0} \cdot s_{n}^{n-1}$;

(4) $\operatorname{vol}(\alpha+\beta)^{1 / n}=\operatorname{vol}(\alpha)^{1 / n}+\operatorname{vol}(\beta)^{1 / n}$;

(5) $\alpha$ and $\beta$ are proportional;

(6) $\alpha^{n-1}$ and $\beta^{n-1}$ are proportional.

As a consequence, the map $\gamma \mapsto \gamma^{n-1}$ is injective from the big and nef cone $\overline{\mathcal{K}} \cap \mathcal{E}^{\circ}$ to the movable cone $\overline{\mathcal{M}}$. 


\section{TEISSIER'S PROPORTIONALITY PROBLEM}

Proof. For a projective algebraic manifold, the usual Khovanskii-Teissier inequalities imply that

$$
s_{k}^{2} \geqslant s_{k-1} \cdot s_{k+1} \quad \text { for } 1 \leqslant k \leqslant n-1
$$

if $\alpha$ and $\beta$ are two nef divisor classes. We remark that it also holds true if $\alpha$ and $\beta$ are two transcendental nef classes on a compact Kähler manifold (see, for example, [Dem93, Section 5], [DP03, Proposition 2.5], [DN06, Theorems A and C] or [Gro90, Section 2]).

For the reader's convenience, we include a proof here (see also [Cao13, Proposition 6.2.1]), which follows from the result on mixed Hodge-Riemann bilinear relations for compact Kähler manifolds [DN06] as in the projective algebraic manifold situation. To this end, let $\omega_{1}, \ldots, \omega_{n-2}$ be $n-2$ Kähler classes on $X$. Consider the following quadratic form $Q$ on $H_{B C}^{1,1}(X, \mathbb{R})$ :

$$
Q(\lambda, \mu):=\int_{X} \lambda \wedge \mu \wedge \omega_{1} \wedge \cdots \wedge \omega_{n-2}
$$

According to [DN06, Theorems A and C], the form $Q$ is of signature $\left(1, h^{1,1}\right)$. For any $\alpha, \beta \in \overline{\mathcal{K}}$ and $t \in \mathbb{R}$, consider the function

$$
Q(\alpha+t \beta, \alpha+t \beta)
$$

As a function of $t$, we claim that $Q(\alpha+t \beta, \alpha+t \beta)=0$ has at least one real solution. We only need to consider the case when $\alpha$ and $\beta$ are linearly independent and thus, $\alpha$ and $\beta$ span a 2-dimensional subspace of $H_{B C}^{1,1}(X, \mathbb{R})$. In view of the signature of $Q$, the function cannot be positive on this 2-dimensional subspace. Now our claim easily follows from this. The existence of real solutions is equivalent to

$$
\left(\int_{X} \alpha \wedge \beta \wedge \omega_{1} \wedge \cdots \wedge \omega_{n-2}\right)^{2} \geqslant\left(\int_{X} \alpha^{2} \wedge \omega_{1} \wedge \cdots \wedge \omega_{n-2}\right) \cdot\left(\int_{X} \beta^{2} \wedge \omega_{1} \wedge \cdots \wedge \omega_{n-2}\right) .
$$

Since $\omega_{1}, \ldots, \omega_{n-2}$ are arbitrary, choosing appropriate $\omega_{i}$ and then taking limits, we obtain the inequalities (2.1) for any two transcendental nef classes.

We begin to prove the main result. Indeed, it is easy to see the equivalence of statements (1)-(4) (see, for example, [Cut15]). Since $\alpha, \beta \in \overline{\mathcal{K}} \cap \mathcal{E}^{\circ}$, it is clear that $s_{k}>0$ for $0 \leqslant k \leqslant n$.

We first prove the equivalence (1) $\Leftrightarrow(3)$. It is trivial that (1) implies (3). On the other hand, the inequalities (2.1) imply

$$
\begin{aligned}
\frac{s_{n-1}}{s_{0}} & =\frac{s_{n-1}}{s_{n-2}} \cdot \frac{s_{n-2}}{s_{n-3}} \cdots \frac{s_{1}}{s_{0}} \\
& \geqslant \frac{s_{n-1}}{s_{n-2}} \cdot \frac{s_{n-2}}{s_{n-3}} \cdots\left(\frac{s_{2}}{s_{1}}\right)^{2} \geqslant \cdots \geqslant\left(\frac{s_{n}}{s_{n-1}}\right)^{n-1} .
\end{aligned}
$$

Thus, if statement (3) holds, then all the above inequalities must be equalities, hence statement (1) holds.

Next, let us prove the equivalence $(1) \Leftrightarrow(2)$. This also follows from (2.1). By (2.1), we have

$$
\left(\frac{s_{k}}{s_{k-1}}\right)^{n-k} \cdots\left(\frac{s_{1}}{s_{0}}\right)^{n-k} \geqslant\left(\frac{s_{n}}{s_{n-1}}\right)^{k} \cdots\left(\frac{s_{k+1}}{s_{k}}\right)^{k}
$$

which clearly implies the equivalence $(1) \Leftrightarrow(2)$.

Now, we prove the equivalence $(2) \Leftrightarrow(4)$. The inequality $(2.2)$ can be rewritten as

$$
s_{k}^{n} \geqslant s_{0}^{n-k} \cdot s_{n}^{k} \text { for } 0 \leqslant k \leqslant n .
$$




\section{J. Fu AND J. XIAO}

These inequalities yield

$$
\begin{aligned}
\operatorname{vol}(\alpha+\beta) & =(\alpha+\beta)^{n}=\sum_{k} \frac{n !}{k !(n-k) !} s_{k} \\
& \geqslant \sum_{k} \frac{n !}{k !(n-k) !} s_{0}^{n-k / n} \cdot s_{n}^{k / n}=\left(\operatorname{vol}(\alpha)^{1 / n}+\operatorname{vol}(\beta)^{1 / n}\right)^{n}
\end{aligned}
$$

which clearly implies the equivalence $(2) \Leftrightarrow(4)$.

Next, we prove the equivalence $(3) \Leftrightarrow(5)$. The implication $(5) \Rightarrow(3)$ is trivial. The real difficulty is to prove the implication $(3) \Rightarrow(5)$. The proof is inspired by our previous work [FX14]. To make the proof of the implication $(3) \Rightarrow(5)$ clear, let us first give a sketch of the proof. Without loss of generality, we assume $\operatorname{vol}(\alpha)=\operatorname{vol}(\beta)$ in the following; we then need to prove that the classes $\alpha$ and $\beta$ are equal. If statement (3) holds, we construct two equal positive $(1,1)$ currents in $\alpha$ and $\beta$, respectively. To prove this, we first construct two positive $(1,1)$-currents in $\alpha$ and $\beta$ respectively, which are equal on a Zariski-open set. The construction heavily depends on the main theorem in [BEGZ10], which solves Monge-Ampère equations in big cohomology classes. Then, by the support theorem of currents, the difference of these two currents can only be a combination of some prime divisors. By showing that all the coefficients in the combination vanish, we deduce that these two currents are equal. Hence, this implies statement (5). All is all, the key elements in the proof of the implication $(3) \Rightarrow(5)$ are solving Monge-Ampère equations in big and nef cohomology classes and using some basic facts in pluripotential theory. In the following, we carry out the details.

For simplicity, we will use the same symbol $\alpha$ (respectively, $\beta$ ) to denote a smooth representative in the cohomology class $\alpha$ (respectively, $\beta$ ). Fix a Kähler metric $\omega$ and a smooth volume form $\Phi$ with $\int_{X} \Phi=1$.

As a starting point, to see how the above ideas work, we first give a proof of the implication $(3) \Rightarrow(5)$ when both $\alpha$ and $\beta$ are Kähler classes. In this simple case, we can easily construct two equal Kähler metrics. By [Yau78], we can solve the following two Monge-Ampère equations:

$$
\begin{aligned}
& (\alpha+i \partial \bar{\partial} \varphi)^{n}=c_{\alpha} \Phi, \\
& (\beta+i \partial \bar{\partial} \psi)^{n}=c_{\beta} \Phi,
\end{aligned}
$$

where $\alpha_{\varphi}:=\alpha+i \partial \bar{\partial} \varphi$ and $\beta_{\psi}:=\beta+i \partial \bar{\partial} \psi$ are two Kähler metrics and $c_{\alpha}=c_{\beta}$ by our assumption $\operatorname{vol}(\alpha)=\operatorname{vol}(\beta)$. We claim that assumption (3) implies $\alpha_{\varphi}=\beta_{\psi}$. To this end, let us consider the two $(n-1, n-1)$-forms $\alpha_{\varphi}^{n-1}$ and $\beta_{\psi}^{n-1}$. We write

$$
\alpha_{\varphi}^{n-1}=\beta_{\psi}^{n-1}+\Theta
$$

for some $(n-1, n-1)$-form $\Theta$. If we can prove $\Theta=0$, then we get $\alpha_{\varphi}=\beta_{\psi}$. We consider the $(1,1)$-form or $(n-1, n-1)$-form as a matrix; then we have the equality

$$
\frac{\operatorname{det} \alpha_{\varphi}^{n-1}}{\operatorname{det} \beta_{\psi}^{n-1}}=\frac{\operatorname{det}\left(\beta_{\psi}^{n-1}+\Theta\right)}{\operatorname{det} \beta_{\psi}^{n-1}}=\left(\frac{\operatorname{det} \alpha_{\varphi}}{\operatorname{det} \beta_{\psi}}\right)^{n-1}=1 .
$$

Note that we have the elementary pointwise inequality

$$
1=\left(\frac{\operatorname{det}\left(\beta_{\psi}^{n-1}+\Theta\right)}{\operatorname{det} \beta_{\psi}^{n-1}}\right)^{1 / n} \leqslant 1+\frac{1}{n} \sum_{i j}\left(\beta_{\psi}^{n-1}\right)^{i \bar{j}} \Theta_{i \bar{j}},
$$

where $\left[\left(\beta_{\psi}^{n-1}\right)^{i \bar{j}}\right]$ is the inverse of the matrix $\beta_{\psi}^{n-1}$. After multiplying both sides of (2.3) by $\beta_{\psi}^{n}$, 


\section{TEISSIER'S PROPORTIONALITY PROBLEM}

it is easy to see that $(2.3)$ is equivalent to

$$
\beta_{\psi}^{n} \leqslant \beta_{\psi}^{n}+\beta_{\psi} \wedge \Theta
$$

Thus $\beta_{\psi} \wedge \Theta$ is a positive $(n, n)$-form on $X$. By assumption (3), we have

$$
\int_{X} \beta_{\psi} \wedge \Theta=\int_{X} \beta_{\psi} \wedge\left(\alpha_{\varphi}^{n-1}-\beta_{\psi}^{n-1}\right)=0
$$

So the inequality (2.3) must be an equality everywhere, which forces $\Theta=0$. This finishes the proof when $\alpha$ and $\beta$ are Kähler classes.

Next, we begin the proof when $\alpha$ and $\beta$ are big and nef classes. Comparing with the proof of the Kähler classes case, we see that we need to solve a family of Monge-Ampère equations and analyze the behavior of the family of solutions.

By [BEGZ10, Theorem C], we can solve the following two degenerate complex Monge-Ampère equations:

$$
\begin{aligned}
& \left\langle(\alpha+i \partial \bar{\partial} \varphi)^{n}\right\rangle=c_{\alpha, 0} \Phi, \\
& \left\langle(\beta+i \partial \bar{\partial} \psi)^{n}\right\rangle=c_{\beta, 0} \Phi,
\end{aligned}
$$

where $\langle-\rangle$ denotes the non-pluripolar product of positive currents and

$$
c_{\alpha, 0}=\operatorname{vol}(\alpha)=\operatorname{vol}(\beta)=c_{\beta, 0} .
$$

Moreover, $\varphi$ (respectively, $\psi$ ) has minimal singularities and is smooth on the ample locus $\operatorname{Amp}(\alpha)$ (respectively, $\operatorname{Amp}(\beta)$ ), which is a Zariski-open set of $X$ depending only on the cohomology class of $\alpha$ (respectively, $\beta$ ).

Let us first briefly recall how the solutions $\varphi$ and $\psi$ are obtained, which is needed in our proof. Indeed, based on Yau's seminal work [Yau78] on the Calabi conjecture, the above two degenerate complex Monge-Ampère equations can be solved by approximations. By Yau's theorem, for $0<t<1$, we can solve the following two families of Monge-Ampère equations:

$$
\begin{aligned}
\left(\alpha+t \omega+i \partial \bar{\partial} \varphi_{t}\right)^{n} & =c_{\alpha, t} \Phi, \\
\left(\beta+t \omega+i \partial \bar{\partial} \psi_{t}\right)^{n} & =c_{\beta, t} \Phi,
\end{aligned}
$$

where $c_{\alpha, t}=\int_{X}(\alpha+t \omega)^{n}, c_{\beta, t}=\int_{X}(\beta+t \omega)^{n}$ and $\sup _{X} \varphi_{t}=\sup _{X} \psi_{t}=0$. Set

$$
\alpha_{t}:=\alpha+t \omega+i \partial \bar{\partial} \varphi_{t} \quad \text { and } \quad \beta_{t}:=\beta+t \omega+i \partial \bar{\partial} \psi_{t} .
$$

We consider the limits of $\alpha_{t}$ and $\beta_{t}$ as $t$ tends to zero. By the basic properties of plurisubharmonic functions, the family of solutions $\left\{\varphi_{t}\right\}_{t}$ (respectively, $\left.\left\{\psi_{t}\right\}_{t}\right)$ is compact in the $L^{1}(X)$-topology, since

$$
\sup _{X} \varphi_{t}=\sup _{X} \psi_{t}=0
$$

Thus, there exists a convergent subsequence which we still denote by the same symbol $\left\{\varphi_{t}\right\}_{t}$ (respectively, $\left\{\psi_{t}\right\}_{t}$ ), and there exists an $\alpha$-psh function $\varphi$ (respectively, a $\beta$-psh function $\psi$ ) such that, when $t$ tends to zero, we have the following limits in the sense of currents on $X$ :

$$
\alpha_{t} \rightarrow \alpha+i \partial \bar{\partial} \varphi
$$

and

$$
\beta_{t} \rightarrow \beta+i \partial \bar{\partial} \psi
$$

Moreover, by the theory developed in [BEGZ10] and the basic estimates in [Yau78], the solution $\varphi_{t}$ (respectively, $\left.\psi_{t}\right)$ is compact in $C_{\mathrm{loc}}^{\infty}(\operatorname{Amp}(\alpha))$ (respectively, $C_{\mathrm{loc}}^{\infty}(\operatorname{Amp}(\beta))$ ). Therefore, 


\section{J. Fu AND J. XIAO}

there exist convergent subsequences such that the convergences (2.4) and (2.5) are in the topologies of $C_{\mathrm{loc}}^{\infty}(\operatorname{Amp}(\alpha))$ and $C_{\mathrm{loc}}^{\infty}(\operatorname{Amp}(\beta))$, respectively. Hence, $\varphi$ (respectively, $\psi$ ) is smooth on $\operatorname{Amp}(\alpha)$ (respectively, $\operatorname{Amp}(\beta))$. Moreover, since $\Phi$ is a smooth volume form, $\alpha+i \partial \bar{\partial} \varphi$ (respectively, $\beta+i \partial \bar{\partial} \psi)$ must be a Kähler metric on $\operatorname{Amp}(\alpha)$ (respectively, $\operatorname{Amp}(\beta))$.

Denote the Zariski-open set $\operatorname{Amp}(\alpha) \cap \operatorname{Amp}(\beta)$ by $\operatorname{Amp}(\alpha, \beta)$, and set

$$
\alpha_{0}:=\alpha+i \partial \bar{\partial} \varphi, \quad \beta_{0}:=\beta+i \partial \bar{\partial} \psi .
$$

We first show that $\alpha_{0}=\beta_{0}$ on $\operatorname{Amp}(\alpha, \beta)$. Let $c_{t}=c_{\alpha, t} / c_{\beta, t}$. By our assumption $\operatorname{vol}(\alpha)=\operatorname{vol}(\beta)$, it is clear that

$$
\lim _{t \rightarrow 0} c_{t}=1
$$

Assume $\alpha^{n-1}=\beta^{n-1}+\Theta(\alpha, \beta)$ for some smooth $(n-1, n-1)$-form $\Theta(\alpha, \beta)$. Then

$$
\alpha_{t}^{n-1}=\beta_{t}^{n-1}+\Theta_{t}
$$

for some smooth $(n-1, n-1)$-form $\Theta_{t}$. Pointwise, $\alpha_{t}, \beta_{t}, \alpha_{t}^{n-1}, \beta_{t}^{n-1}$ and $\Theta_{t}$ can be viewed as matrices. In this sense, we have

$$
\frac{\operatorname{det} \alpha_{t}^{n-1}}{\operatorname{det} \beta_{t}^{n-1}}=\left(\frac{\operatorname{det} \alpha_{t}}{\operatorname{det} \beta_{t}}\right)^{n-1}
$$

Hence, as in the Kähler classes case, we have

$$
\begin{aligned}
c_{t}^{n-1 / n} & =\left(\frac{\operatorname{det} \alpha_{t}^{n-1}}{\operatorname{det} \beta_{t}^{n-1}}\right)^{1 / n}=\left(\frac{\operatorname{det}\left(\beta_{t}^{n-1}+\Theta_{t}\right)}{\operatorname{det} \beta_{t}^{n-1}}\right)^{1 / n} \\
& \leqslant 1+\frac{1}{n} \sum_{i j}\left(\beta_{t}^{n-1}\right)^{i \bar{j}}\left(\Theta_{t}\right)_{i \bar{j}},
\end{aligned}
$$

where the matrix $\left[\left(\beta_{t}^{n-1}\right)^{i \bar{j}}\right]$ is the inverse of $\beta_{t}^{n-1}$. Equivalently, multiplying both sides of (2.7) by $\beta_{t}^{n}$, we get

$$
c_{t}^{n-1 / n} \beta_{t}^{n} \leqslant \beta_{t}^{n}+\beta_{t} \wedge \Theta_{t}
$$

Note that we have

$$
\beta_{t} \wedge \Theta_{t}=\alpha_{t}^{n-1} \wedge \beta_{t}-\beta_{t}^{n} .
$$

Consider $\left\{\alpha_{t}^{n-1} \wedge \beta_{t}\right\}_{t}$ (respectively, $\left\{\beta_{t}^{n}\right\}_{t}$ ) as a family of positive measures; then it is of bounded mass. Thus there exist convergent subsequences, which we still denote by $\left\{\alpha_{t}^{n-1} \wedge \beta_{t}\right\}_{t}$ and $\left\{\beta_{t}^{n}\right\}_{t}$, and positive measures $\mu_{1}$ and $\mu_{2}$ such that

$$
\begin{aligned}
\alpha_{t}^{n-1} \wedge \beta_{t} & \rightarrow \mu_{1}, \\
\beta_{t}^{n} & \rightarrow \mu_{2},
\end{aligned}
$$

in the sense of measures. If we write $\mu=\mu_{1}-\mu_{2}$, then we get $\beta_{t} \wedge \Theta_{t} \rightarrow \mu$.

We claim that $\mu$ is a zero measure. It is not hard to see from (2.6) and (2.8) that $\mu$ is a positive measure on $X$. Indeed, let $f$ be any positive continuous function over $X$; we have

$$
\int_{X} f \mu=\lim _{t \rightarrow 0} \int_{X} f\left(\beta_{t} \wedge \Theta_{t}\right) \geqslant \liminf _{t \rightarrow 0} \int_{X} f\left(c_{t}^{n-1 / n} \beta_{t}^{n}-\beta_{t}^{n}\right)=0 .
$$

Meanwhile, assumption (3) implies

$$
\int_{X} \mu=\lim _{t \rightarrow 0} \int_{X}\left(\beta_{t} \wedge \alpha_{t}^{n-1}-\beta_{t}^{n}\right)=\int_{X}\left(\beta \wedge \alpha^{n-1}-\beta^{n}\right)=0 .
$$




\section{TEISSIER'S PROPORTIONALITY PROBLEM}

Hence $\mu$ must be a zero measure. In particular, since $\operatorname{Amp}(\alpha, \beta)$ is a Zariski-open set (thus a Borel-measurable set), we have

$$
\beta_{t} \wedge \Theta_{t} \rightarrow 0
$$

in the sense of measures on $\operatorname{Amp}(\alpha, \beta)$.

Using the convergences (2.4) and (2.5) in the topologies of $C_{\mathrm{loc}}^{\infty}(\operatorname{Amp}(\alpha))$ and $C_{\mathrm{loc}}^{\infty}(\operatorname{Amp}(\beta))$, respectively, it is clear that there exists some smooth form $\Theta_{0}$ which is only defined on $\operatorname{Amp}(\alpha, \beta)$, such that $\Theta_{t} \rightarrow \Theta_{0}$ in the topology of $C_{\text {loc }}^{\infty}(\operatorname{Amp}(\alpha, \beta))$. This implies that in the same topology

$$
\beta_{t} \wedge \Theta_{t} \rightarrow \beta_{0} \wedge \Theta_{0}
$$

Combining (2.9) and (2.10) and using uniqueness of the limit, we obtain

$$
\beta_{0} \wedge \Theta_{0}=0
$$

on $\operatorname{Amp}(\alpha, \beta)$. This equality implies that if we take the limits on $\operatorname{Amp}(\alpha, \beta)$ of both sides of (2.7), we have

$$
1=\left(\frac{\operatorname{det} \alpha_{0}^{n-1}}{\operatorname{det} \beta_{0}^{n-1}}\right)^{1 / n}=\left(\frac{\operatorname{det}\left(\beta_{0}^{n-1}+\Theta_{0}\right)}{\operatorname{det} \beta_{0}^{n-1}}\right)^{1 / n} \leqslant 1+\frac{1}{n} \sum_{i, j}\left(\beta_{0}^{n-1}\right)^{i \bar{j}}\left(\Theta_{0}\right)_{i \bar{j}}=1 .
$$

This forces $\Theta_{0}=0$, and hence $\alpha_{0}^{n-1}=\beta_{0}^{n-1}$ on $\operatorname{Amp}(\alpha, \beta)$. Since $\alpha_{0}$ and $\beta_{0}$ are Kähler metrics, we have $\alpha_{0}=\beta_{0}$ on $\operatorname{Amp}(\alpha, \beta)$.

We claim that $\alpha_{0}=\beta_{0}$ on $X$. Before going on, we need the following two lemmas.

Lemma 2.2 (see [Dem12, Chapter III]). Let $T$ be a $d$-closed $(p, p)$-current. Suppose that the support supp $T$ of $T$ is contained in an analytic subset $A$. If $\operatorname{dim} A<n-p$, then $T=0$; if $T$ is of order zero and $A$ is of pure dimension $n-p$ with $(n-p)$-dimensional irreducible components $A_{1}, \ldots, A_{k}$, then $T=\sum c_{j}\left[A_{j}\right]$ with $c_{j} \in \mathbb{C}$.

Lemma 2.3 (see [Bou04, Propositions 3.2 and 3.6]). Let $\alpha$ be a big and nef class, and let $T_{\min }$ be a positive current in $\alpha$ with minimal singularities. Then the Lelong number $\nu\left(T_{\min }, x\right)$ is 0 for any point $x \in X$.

It is clear that $S:=X \backslash \operatorname{Amp}(\alpha, \beta)$ is a proper analytic subset of $X$. Let $T=\alpha_{0}-\beta_{0}$; then $T$ is a real $d$-closed $(1,1)$-current and $\operatorname{supp} T \subset S$. If $\operatorname{codim} S \geqslant 2$, then $T=0$ according to Lemma 2.2. This implies $\alpha_{0}=\beta_{0}$ on $X$. If codim $S=1$ and $S$ has only irreducible components $D_{1}, \ldots, D_{k}$ of pure dimension one, then Lemma 2.2 implies

$$
\alpha_{0}-\beta_{0}=\sum c_{j}\left[D_{j}\right] .
$$

If $\operatorname{codim} S=1$ and $S$ has also components of codimension more than one, we just repeat the proof of Lemma 2.2 as in [Dem12] (for more details, see, for example, [FX14]), and still get $\alpha_{0}-\beta_{0}=\sum c_{j}\left[D_{j}\right]$. Since $\alpha_{0}$ and $\beta_{0}$ are real, all $c_{j}$ can be chosen to be real numbers. If there exists at least one $c_{j}>0$, we write this equality as

$$
\alpha_{0}-\sum c_{j^{\prime}}\left[D_{j^{\prime}}\right]=\beta_{0}+\sum c_{j^{\prime \prime}}\left[D_{j^{\prime \prime}}\right]
$$

with $c_{j^{\prime}} \leqslant 0$ and $c_{j^{\prime \prime}}>0$.

Fix a $j^{\prime \prime}$, which we denote by $j_{0}^{\prime \prime}$. We take a generic point $x \in D_{j_{0}^{\prime \prime}}$; for example, we can take such a point $x$ with $\nu\left(\left[D_{j_{0}^{\prime \prime}}\right], x\right)=1$ and $x \notin \cup_{j \neq j_{0}^{\prime \prime}} D_{j}$. Then, taking the Lelong number at the point $x$ on both sides of (2.12), we have

$$
\nu\left(\alpha_{0}, x\right)-\sum c_{j^{\prime}} \nu\left(\left[D_{j^{\prime}}\right], x\right)=\nu\left(\beta_{0}, x\right)+\sum c_{j^{\prime \prime}} \nu\left(\left[D_{j^{\prime \prime}}\right], x\right) .
$$




\section{J. FU AND J. XIAO}

Since $\alpha_{0}$ and $\beta_{0}$ are positive currents with minimal singularities in big and nef classes, Lemma 2.3 tells us that $\nu\left(\alpha_{0}, x\right)=0$ and $\nu\left(\beta_{0}, x\right)=0$. The property of $x$ also implies $\nu\left(\left[D_{j^{\prime}}\right], x\right)=0$ and $\nu\left(\left[D_{j^{\prime \prime}}\right], x\right)=0$ for all $j^{\prime}$ and all $j^{\prime \prime} \neq j_{0}^{\prime \prime}$. All these force $c_{j_{0}^{\prime \prime}}=0$, which contradicts our assumption $c_{j_{0}^{\prime \prime}}>0$. Thus, we have

$$
\alpha_{0}-\sum c_{j^{\prime}}\left[D_{j^{\prime}}\right]=\beta_{0} .
$$

By the same reason, we can also prove $c_{j^{\prime}}=0$. Hence, we have finished the proof of $\alpha_{0}=\beta_{0}$ over $X$ and the proof of the implication $(3) \Rightarrow(5)$.

The implication $(5) \Rightarrow(6)$ is trivial. For the implication $(6) \Rightarrow(3)$, suppose $\alpha^{n-1}=c \beta^{n-1}$ for some $c>0$; then we have

$$
\alpha^{n}=c \beta^{n-1} \cdot \alpha \geqslant c\left(\beta^{n}\right)^{n-1 / n}\left(\alpha^{n}\right)^{1 / n}
$$

and

$$
\alpha^{n-1} \cdot \beta=c \beta^{n} \geqslant\left(\alpha^{n}\right)^{n-1 / n}\left(\beta^{n}\right)^{1 / n} .
$$

This yields $\left(\alpha^{n}\right)^{n-1 / n}=c\left(\beta^{n}\right)^{n-1 / n}$. As a consequence, we get

$$
\alpha^{n-1} \cdot \beta=c \beta^{n}=\left(\alpha^{n}\right)^{n-1 / n}\left(\beta^{n}\right)^{1 / n},
$$

which is just statement (3). By the implication $(3) \Rightarrow(5)$, we then have the equivalences $(5) \Leftrightarrow$ $(6) \Leftrightarrow(3)$.

Summarizing all the above arguments, we have finished the proof of the equivalence of statements (1)-(6). In particular, we get that the map $\gamma \mapsto \gamma^{n-1}$ is injective from the big and nef cone $\overline{\mathcal{K}} \cap \mathcal{E}^{\circ}$ to the movable cone $\overline{\mathcal{M}}$.

\section{ACKNOWLEDGEMENTS}

Jian Xiao would like to thank Professor Jean-Pierre Demailly for his interests and encouragement.

\section{REFERENCES}

BDPP13 S. Boucksom, J.-P. Demailly, M. Păun and T. Peternell, The pseudo-effective cone of a compact Kähler manifold and varieties of negative Kodaira dimension, J. Algebraic Geom. 22 (2013), no. 2, 201-248; doi:10.1090/S1056-3911-2012-00574-8.

BEGZ10 S. Boucksom, P. Eyssidieux, V. Guedj and A. Zeriahi, Monge-Ampère equations in big cohomology classes, Acta Math. 205 (2010), no. 2, 199-262; doi:10.1007/s11511-010-0054-7.

BFJ09 S. Boucksom, C. Favre and M. Jonsson, Differentiability of volumes of divisors and a problem of Teissier, J. Algebraic Geom. 18 (2009), no. 2, 279-308; doi:10.1090/S1056-3911-08-00490-6.

Bou04 S. Boucksom, Divisorial Zariski decompositions on compact complex manifolds, Ann. Sci. École Norm. Sup. (4) 37 (2004), no. 1, 45-76; doi:10.1016/j.ansens.2003.04.002.

Cao13 J. Cao, Théorèmes d'annulation et théorèmes de structure sur les variétès kähleriennes compactes, Ph.D. Thesis, Université Joseph-Fourier-Grenoble I, 2013, available at http://www. theses.fr/2013GRENM017.pdf.

Cut15 S. D. Cutkosky, Teissier's problem on inequalities of nef divisors, J. Algebra Appl. 14 (2015), no. 9, 1540002; doi:10.1142/S0219498815400022.

Dem93 J.-P. Demailly, A numerical criterion for very ample line bundles, J. Differential Geom. 37 (1993), no. 2, 323-374; doi:10.4310/jdg/1214453680. 


\section{TEISSIER'S PROPORTIONALITY PROBLEM}

Dem12 Complex analytic and differential geometry, online book, Institut Fourier, Grenoble, 2012, available at https://www-fourier.ujf-grenoble.fr/ demailly/manuscripts/ agbook.pdf.

DN06 T.-C. Dinh and V.-A. Nguyên, The mixed Hodge-Riemann bilinear relations for compact Kähler manifolds, Geom. Funct. Anal. 16 (2006), no. 4, 838-849; doi:10.1007/s00039-006-0572-9.

DP03 J.-P. Demailly and T. Peternell, A Kawamata-Viehweg vanishing theorem on compact Kähler manifolds, J. Differential Geom. 63 (2003), no. 2, 231-277; doi:10.4310/jdg/1090426678.

FX14 J. Fu and J. Xiao, Relations between the Kähler cone and the balanced cone of a Kähler manifold, Adv. Math. 263 (2014), 230-252; doi:10.1016/j.aim.2014.06.018.

Gro90 M. Gromov, Convex sets and Kähler manifolds, in Advances in Differential Geometry and Topology (World Sci. Publ., Teaneck, NJ, 1990), 1-38; doi:10.1142/9789814439381_0001.

LX16 B. Lehmann and J. Xiao, Convexity and Zariski decomposition structure, Geom. Funct. Anal. 26 (2016), no. 4, 1135-1189; doi:10.1007/s00039-016-0384-5.

Pop16 D. Popovici, Sufficient bigness criterion for differences of two nef classes, Math. Ann. 364 (2016), no. 1-2,649-655; doi:10.1007/s00208-015-1230-z.

Tei82 B. Teissier, Bonnesen-type inequalities in algebraic geometry. I. Introduction to the problem, Seminar on Differential Geometry, Ann. of Math. Stud., vol. 102 (Princeton Univ. Press, Princeton, N.J., 1982), 85-105.

Tei88_- Monômes, volumes et multiplicités, in Introduction à la théorie des singularités, II, Travaux en Cours, vol. 37 (Hermann, Paris, 1988), 127-141.

Xia15 J. Xiao, Weak transcendental holomorphic Morse inequalities on compact Kähler manifolds, Ann. Inst. Fourier (Grenoble) 65 (2015), no. 3, 1367-1379; doi:10.5802/aif. 2959.

Yau78 S.T. Yau, On the Ricci curvature of a compact Kähler manifold and the complex Monge-Ampère equation. I, Comm. Pure Appl. Math. 31 (1978), no. 3, 339-411; doi:10.1002/cpa.3160310304.

Jixiang $\mathrm{Fu}$ majxfu@fudan.edu.cn

Institute of Mathematics, Fudan University, 200433 Shanghai, China

Jian Xiao jianxiao@math.northwestern.edu

Department of Mathematics, Northwestern University, Evanston, IL 60208, USA

Current address: Department of Mathematical Sciences and Yau Mathematical Sciences Center, Tsinghua University, Beijing 100084, China 